\title{
On Categorical Time Series Models with Covariates
}

\author{
Konstantinos Fokianos* $\quad$ Lionel Truquet ${ }^{\dagger}$
}

First version: October 2017

Second version: July 2018

\begin{abstract}
We study the problem of stationarity and ergodicity for autoregressive multinomial logistic time series models which possibly include a latent process and are defined by a GARCH-type recursive equation. We improve considerably upon the existing conditions about stationarity and ergodicity of those models. Proofs are based on theory developed for chains with complete connections. A useful coupling technique is employed for studying ergodicity of infinite order finite-state stochastic processes which generalize finite-state Markov chains. Furthermore, for the case of finite order Markov chains, we discuss ergodicity properties of a model which includes strongly exogenous but not necessarily bounded covariates.
\end{abstract}

2010 Mathematics Subject Classification: Primary 62M10; secondary 60G10, 60B12.

Keywords and Phrases: autoregression, categorical data, chains with complete connection, coupling, covariates, ergodicity, Markov chains.

\footnotetext{
${ }^{*}$ Lancaster University, Department of Mathematics \& Statistics, Fylde College, Lancaster, LA1 4YF, United Kingdom Email: k.fokianos@lancaster.ac.uk.

†UMR 9194 CNRS CREST, ENSAI, Campus de Ker-Lann, rue Blaise Pascal, BP 37203, 35172 Bruz cedex, France. Email: lionel.truquet@ensai.fr.
} 


\section{Introduction}

The goal of this article is to improve upon theoretical properties of regression based models for the analysis of categorical time series that might include some covariates which are not necessarily bounded. Binary time series are particular cases of a categorical time series and the results we obtain apply to logistic autoregressive models. The conditional distribution of a categorical time series given its past is multinomial which obviously belongs to the multivariate exponential family of distributions. As such, the theory of generalized linear models, see McCullagh and Nelder (1989), can be applied for modeling different types of categorical data; nominal, interval and scale. We will be mostly concerned with nominal data and therefore the multinomial logistic model is the natural candidate for model fitting; see Fahrmeir and Tutz (2001) and Kedem and Fokianos (2002), among other references, for further discussion on modeling issues regarding categorical data. We emphasize that finite state Markov chains provide a simple but prominent model of a categorical time series where lagged values of the response affect the determination of its future states. However, Markov modeling in the context of categorical time series, poses challenging problems. Indeed, as the order of the Markov chain increases so does the number of free parameters; in fact, the number of free parameters increases exponentially fast. Furthermore, the Markovian property requires simultaneous specification of the dynamics of the response and any possible covariates observed jointly; such a specification might not be possible, in general.

We will be studying models for binary and, more generally, categorical time series, which are of infinite order or they are driven by a latent process or a feedback mechanism. This type of models is quite analogous to GARCH models -see Bollerslev (1986)- but they are defined in terms of conditional log-odds instead of conditional variances. In particular, feedback models make possible low dimensional parametrization, yet they can accommodate quite complicated data structures. Examples of feedback models, in the context of binary and categorical time series have been studied recently by Moysiadis and Fokianos (2014) and Fokianos and Moysiadis (2017), among others. We will discuss their results and we will compare them with our findings. Models and inference for binary time series are research topics that have been studied by several authors; see Kedem (1980) for an early treatment. Regression modeling, in this context, has been studied by Cox (1981), Stern and Coe (1984), and Slud and Kedem (1994), among others; see also Kedem and Fokianos (2002, Ch. 2-3) for other early references. Recently, binary time series data have been increasingly popular in various financial applications (Breen et al. (1989), Butler and Malaikah (1992), Christoffersen and Diebold (2006), Christoffersen et al. (2007), Startz (2008), Nyberg (2010, 2011, 
2013), Kauppi (2012) and Wu and Cui (2014)), but also to other scientific fields. Previous results related to theoretical properties of binary time series models were given by de Jong and Woutersen (2011).

Related work on categorical time series has been reported by Fahrmeir and Kaufmann (1987), Kaufmann (1987), Fokianos and Kedem (2003) and Russell and Engle (1998, 2005) who proposed a categorical time series model for financial transactions data. Alternative classes of models are based on the probit link function. Such autoregressive models have been considered by Zeger and Qaqish (1988), Rydberg and Shephard (2003), Kauppi and Saikkonen (2008), among others. Several alternative classes of models for the analysis of categorical data have been studied; see the books by Joe (1997) and MacDonald and Zucchini (1997) and the articles by Biswas and Song (2009) and Weiß (2011).

To prove the theoretical results, we will be assuming a contraction type condition; such conditions are usually employed for the theoretical analysis of time series models. For instance, in the case of count time series models, see Fokianos et al. (2009), Neumann (2011) and Doukhan et al. (2012). However, our work is closely related to the modeling approach suggested by Fokianos and Tjøstheim (2011), because the main idea is, essentially, to employ the so called canonical link process to model the observed data. Note that de Jong and Woutersen (2011) have shown near epoch dependence for a binary time series models but these authors have a different modeling point of view.

Likelihood based inference for the models we study can be developed along the lines of previous references by appealing to the properties of multinomial distribution. The proof of consistency and asymptotic normality for the maximum likelihood estimator is based on standard arguments concerning convergence of the score function and the Hessian matrix. In addition, differentiability properties of multinomial likelihood allow obtaining suitable bounds for higher order derivatives. However, we mention that this work relaxes considerably previous results. For the case of a model with covariates we improve considerably upon Kaufmann (1987) and Kedem and Fokianos (2002, Ch.3) because we avoid any assumptions on the design of covariates. In addition, we show that we obtain ergodicity even in the case of finite order models with unbounded covariates. The study of maximum likelihood estimation requires existence of appropriate moments for the covariate process though. Central limit theorems for the maximum likelihood estimators have been given in the previous references and therefore we do not give any further details.

Section 2 discusses general categorical time series models by allowing the conditional probabilities to depend on the whole past of the series. In addition we will be giving a result about the stationarity and ergodicity of chains with complete connections. These results will be applied to the case of an infinite 
order autoregressive multinomial logistic model. Section 3 discuss models which might include a latent process. The results obtained by Theorem 1 improve the results obtained by Moysiadis and Fokianos (2014) and Fokianos and Moysiadis (2017). Finally, Section 4 discuss inclusion of exogenous covariates to the autoregressive multinomial logistic model of finite order. Theorem 2, which is the main result in this section, proves existence of such processes and their ergodicity.

\section{Time series autoregressive models for categorical data}

\subsection{A general approach}

Let $\mathcal{A}$ be a finite set. For simplicity, we assume that $\mathcal{A}=\{1,2, \ldots, N\}$, where $N$ is a nonnegative integer. Suppose that we observe a process with state space $\mathcal{A}$ and we are interested on modeling its dynamics. For instance, consider modeling of a stock price change ( 0 for no change, 1 for positive change and -1 for a negative change; see Russell and Engle (1998)), sleep state status (see Fokianos and Kedem (2003)), and wage mobility data (see Pamminger and Frühwirth-Schnatter (2010)). Towards this goal, define a $(N-1)$ dimensional vector $Y_{t}=\left(Y_{1 t}, Y_{2 t}, \ldots, Y_{(N-1) t}\right)^{\prime}$, for $1 \leq t \leq n$, such that

$$
Y_{k t}= \begin{cases}1, & \text { if the } k^{\prime} \text { th category is observed at time } t, \\ 0, & \text { otherwise, }\end{cases}
$$

for all $k=1,2, \ldots, N-1$. Throughout this work, consider a stochastic processes $\left(Y_{t}\right)_{t \in \mathbb{Z}}$ adapted to a filtration $\left(\mathcal{F}_{t}\right)_{t \in \mathbb{Z}}$ which is defined through a vector of conditional "success" probabilities, say $p_{t} \equiv\left(p_{1 t}, p_{2 t}, \ldots, p_{(N-1) t}\right)^{\prime}$. In other words

$$
p_{k t}=\mathbb{P}\left(Y_{k t}=1 \mid \mathcal{F}_{t-1}\right), \quad 1 \leq j \leq N-1 .
$$

For the last category $N$, set $Y_{N t}=1-\sum_{k=1}^{N-1} Y_{k t}$ and $p_{N t}=1-\sum_{k=1}^{N-1} p_{k t}$.

There are several possibilities for autoregressive modeling of processes that take values on a finite space. For instance, assuming that $d$ is a vector and $A, B$ matrices of appropriate dimension, consider the following linear model

$$
p_{t}=d+A p_{t-1}+B Y_{t-1}, \quad t \in \mathbb{Z}
$$

which was studied by Russell and Engle (1998) and Qaqish (2003). Model (2) implies quite complex restrictions on the parameters $d, A$ and $B$ because each element of the vector $p_{t}$ has to belong in the interval 
$(0,1)$. Such restrictions become even more involved when a covariate process is included in (2). To avoid such subtle technicalities, we adapt the generalized linear models point of view by considering a canonical link model; see Fokianos and Kedem (2003) for instance. For $k=1,2, \ldots, N-1$, define

$$
\lambda_{k t}=\log \left(p_{k t} / p_{N t}\right)
$$

and suppose that the vector process $\lambda_{t}=\left(\lambda_{1 t}, \ldots, \lambda_{(N-1) t}\right)^{\prime}$ is determined by the infinite order model

$$
\lambda_{t}=g\left(Y_{t-1}, Y_{t-2}, \ldots\right)
$$

where $g$ is a suitably defined function. Then, the process $\left(Y_{t}\right)_{t \in \mathbb{Z}}$ which satisfies (1) and (3), takes its values in the set $E=\left\{e_{1}, e_{2}, \ldots, e_{N-1}, \mathbf{0}\right\}$ where $\left\{e_{1}, \ldots, e_{N-1}\right\}$ is the canonical basis of $\mathbb{R}^{N-1}$ and $\mathbf{0}$ is the null vector

of $\mathbb{R}^{N-1}$. Furthermore, $g: E^{\mathbb{N}} \rightarrow \mathbb{R}^{N-1}$ is a measurable function and the conditional distribution of $Y_{t}$ given its past values $Y_{t-1}^{-} \equiv\left(Y_{t-1}, Y_{t-2}, \ldots\right)$ possibly depends on its infinite past. A useful example of such process is given by the linear process

$$
\lambda_{t}=d+\sum_{j \geq 1} A_{j} Y_{t-j}
$$

where $d$ is a $(N-1)$-dimensional vector and $\left(A_{j}\right)_{j \geq 1}$ is a sequence of $(N-1) \times(N-1)$ matrices. Comparison of (4) to (2) shows that unnecessary restrictions on the unknown coefficients can be circumvented since the vector $\lambda_{t} \in \mathbb{R}^{N-1}$. Furthermore, covariates can be easily included in (4) by including an additional additive term. Other categorical type autoregressive models can be considered but (4) has been used in several applications. In the case that $N=2$, then (4) is a simple logistic regression model which has been studied widely in the literature (see Cox and Snell (1970) for an early reference).

Autoregressive models, as those we consider in this work, are particular examples of a more general class of processes which are called chains with complete connections. Such processes have been widely studied in applied probability; Doeblin and Fortet (1937), Harris (1955) and Iosifescu and Grigorescu (1990). Following the work of Bressaud et al. (1999), we discuss next a coupling technique related to chains with complete connections.

\subsection{Some results about chains with complete connection}

Throughout this section, consider a finite state space $E$. For $x, y \in E^{\mathbb{N}}$ and a positive integer $m$, we write $x \stackrel{m}{=} y$ if $x_{i}=y_{i}$ for $0 \leq i \leq m-1$. Consider a probability kernel $p(\cdot \cdot)$ defined on $\left(E^{\mathbb{N}}, \mathcal{B}\left(E^{\mathbb{N}}\right)\right)$ and taking values on $(E, \mathcal{B}(E))$ which satisfies the following assumption: 
Assumption (A) There exists a sequence $\left(\gamma_{m}\right)_{m \in \mathbb{N}}$ which decreases to zero, as $m \rightarrow \infty$, with $\gamma_{0}<1$ and such that for $a \in E$

$$
\inf _{x, y: x=\underline{m}=y} \frac{p(a \mid x)}{p(a \mid y)} \geq 1-\gamma_{m}
$$

A chain with complete connections is a stationary process satisfying Assumption (A).

For $x \in E^{\mathbb{N}}$, consider the chain $\left(Z_{n}^{x}\right)_{n \in \mathbb{Z}}$ which satisfies that $Z_{-j}^{x}=x_{j}$ for $j \geq 1$ and

$$
\mathbb{P}\left(Z_{n}^{x}=a \mid Z_{n-j}^{x}=z_{j}, j \geq 1\right)=p(a \mid z) \prod_{j=n+1}^{\infty} \mathbb{1}_{z_{j}=x_{j-n}}, \quad n \geq 0 .
$$

In addition, given a real-valued sequence $\left(\gamma_{n}\right)_{n \in \mathbb{N}}$, let the Markov chain $\left(S_{n}^{(\gamma)}\right)_{n \in \mathbb{N}}$ taking values in $\mathbb{N}$ and defined by

$$
\mathbb{P}\left(S_{0}^{(\gamma)}=0\right)=1, \quad \mathbb{P}\left(S_{n+1}^{(\gamma)}=i+1 \mid S_{n}^{(\gamma)}=i\right)=1-\gamma_{i}, \quad \mathbb{P}\left(S_{n+1}^{(\gamma)}=0 \mid S_{n}^{(\gamma)}=i\right)=\gamma_{i}
$$

For $n \geq 1$, define the quantity

$$
\gamma_{n}^{*}=\mathbb{P}\left(S_{n}^{(\gamma)}=0\right),
$$

which plays a crucial rule for evaluating the mixing coefficients of the chain. The following result is given by Bressaud et al. (1999, Prop. 1 and Lemma 1).

Proposition 1. For all $x, y \in E^{\mathbb{N}}$, there is a coupling $\left(\left(U_{n}^{x, y}, V_{n}^{x, y}\right)\right)_{n \in \mathbb{Z}}$ of $\left(Z_{n}^{x}\right)_{n \in \mathbb{Z}}$ and $\left(Z_{n}^{y}\right)_{n \in \mathbb{Z}}$ such that the integer-valued process $\left(T_{n}^{x, y}\right)_{n \in \mathbb{Z}}$ defined by

$$
T_{n}^{x, y}=\inf \left\{m \geq 0: U_{n-m}^{x, y} \neq V_{n-m}^{x, y}\right\},
$$

satisfies

$$
\mathbb{P}\left(S_{n}^{(\gamma)} \geq k\right) \leq \mathbb{P}\left(T_{n}^{x, y} \geq k\right) \forall k \in \mathbb{N}
$$

Proposition 1 is proved by defining iteratively the pair $\left(U_{n}^{x, y}, V_{n}^{x, y}\right)$ using the maximal coupling of the conditional distributions $p\left(\cdot \mid\left(u_{n-j}\right)_{j \geq 1}\right)$ and $p\left(\cdot \mid\left(v_{n-j}\right)_{j \geq 1}\right)$ (i.e the coupling associated to the total variation distance between these conditional distributions). Proposition 1 yields the following corollary (see also Bressaud et al. (1999, Cor. 1) for a specific case of the following result).

Corollary 1. For all $k \geq 1, x, y \in E^{\mathbb{N}}$ and $B \in \mathcal{B}\left(E^{k}\right)$, we have

$$
\left|\mathbb{P}\left(\left(Z_{n}^{x}, \ldots, Z_{n+k}^{x}\right) \in B\right)-\mathbb{P}\left(\left(Z_{n}^{y}, \ldots, Z_{n+k}^{y}\right) \in B\right)\right| \leq \sum_{j=0}^{k}\left(\prod_{m=0}^{j-1}\left(1-\gamma_{m}\right)\right) \gamma_{n+k-j}^{*} .
$$


Proof. Using Proposition 1, we obtain

$$
\begin{aligned}
\left|\mathbb{P}\left(\left(Z_{n}^{x}, \ldots, Z_{n+k}^{x}\right) \in B\right)-\mathbb{P}\left(\left(Z_{n}^{y}, \ldots, Z_{n+k}^{y}\right) \in B\right)\right| & \leq \mathbb{P}\left(\left(U_{n}^{x, y}, \ldots, U_{n+k}^{x, y}\right) \neq\left(V_{n}^{x, y}, \ldots, V_{n+k}^{x, y}\right)\right) \\
& \leq \mathbb{P}\left(T_{n}^{x, y} \leq k\right) .
\end{aligned}
$$

Proposition 1 implies that $\mathbb{P}\left(T_{n}^{x, y} \leq k\right) \leq \mathbb{P}\left(S_{n}^{(\gamma)} \leq k\right)$. The result of the corollary now follows by bounding $\mathbb{P}\left(S_{n}^{(\gamma)} \leq k\right)$ along the lines of the derivation of Bressaud et al. (1999, eq. (4.25)).

As pointed out in Bressaud et al. (1999), if $\lim _{n \rightarrow \infty} \gamma_{n}^{*}=0$, then Corollary 1 implies existence and uniqueness of a stationary chain $\left(Z_{n}\right)_{n \in \mathbb{Z}}$ with complete connections and satisfying Assumption (A). Furthermore, Corollary 1 yields a bound for controlling the $\phi$-mixing coefficients associated with $\left(Z_{n}\right)_{n \in \mathbb{Z}}$. Indeed, recall that for two $\sigma$-algebras $\mathcal{A}$ and $\mathcal{B}$, their $\phi$-mixing coefficients are defined by (see Doukhan (1994), for instance)

$$
\phi(\mathcal{A}, \mathcal{B})=\sup _{(A, B) \in \mathcal{A} \times \mathcal{B}: \mathbb{P}(A)>0}(|\mathbb{P}(B \mid A)-\mathbb{P}(B)|) .
$$

Then, the $\phi$-mixing coefficients of the random sequence $\left(Z_{n}\right)_{n \in \mathbb{Z}}$ are given by

$$
\phi(n)=\phi\left(\mathcal{F}_{-\infty, 0}, \mathcal{F}_{n, \infty}\right)=\sup _{k \in \mathbb{N}} \phi\left(\mathcal{F}_{-\infty, 0}, \mathcal{F}_{n, n+k}\right)
$$

because a Borel set on the infinite product can be approximated by a finite union of cylinder sets.

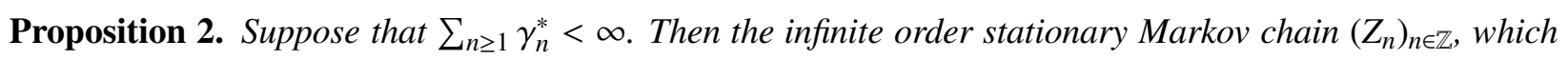
exists by Corollary 1, is $\phi$-mixing with mixing coefficients satisfying $\phi(n) \leq \sum_{j \geq n} \gamma_{j}^{*}$.

Proof. Suppose that $\mu$ denotes the probability distribution of $\left(Z_{i}\right)_{i \leq-1}$. Then

$$
\begin{aligned}
\left|\mathbb{P}\left(\left(Z_{n}^{x}, \ldots, Z_{n+k}^{x}\right) \in B\right)-\mathbb{P}\left(\left(Z_{n}, \ldots, Z_{n+k}\right) \in B\right)\right| & \leq \int\left|\mathbb{P}\left(\left(Z_{n}^{x}, \ldots, Z_{n+k}^{x}\right) \in B\right)-\mathbb{P}\left(\left(Z_{n}^{y}, \ldots, Z_{n+k}^{y}\right) \in B\right)\right| \mu(d y) \\
& \leq \sum_{j=0}^{k}\left(\prod_{m=0}^{j-1}\left(1-\gamma_{m}\right)\right) \gamma_{n+k-j}^{*} .
\end{aligned}
$$

But the last bound does not depend on $x$. Hence, we obtain

$$
\begin{aligned}
\left|\mathbb{P}\left(\left(Z_{n}, \ldots, Z_{n+k}\right) \in B \mid \mathcal{F}_{-\infty, 0}\right)-\mathbb{P}\left(\left(Z_{n}, \ldots, Z_{n+k}\right) \in B\right)\right| & \leq \sum_{j=0}^{k}\left(\prod_{m=0}^{j-1}\left(1-\gamma_{m}\right)\right) \gamma_{n+k-j}^{*} \\
& \leq \sum_{j \geq n} \gamma_{j}^{*} .
\end{aligned}
$$

The last bound, which does not depend on $k$ and $B$, is also an upper bound for $\phi(n)$. 
Remark 1. It has been shown in Bressaud et al. (1999, Prop. 2) that

$$
\sum_{k \geq 1} \gamma_{k}<\infty \Rightarrow \sum_{k \geq 1} \gamma_{k}^{*}<\infty
$$

Moreover, if $\left(\gamma_{m}\right)_{m}$ decreases exponentially, then so does $\left(\gamma_{n}^{*}\right)_{n}$. Hence, the result of Prop. 2 follows again and if $\left(\gamma_{m}\right)_{m}$ decreases to zero exponentially fast then so does $(\phi(n))_{n}$. Note also that the $\phi$-mixing property implies ergodicity of the process; see Bradley (2007, pp. 50-51).

\subsection{Application to categorical time series}

Recall the categorical time series model $\left(Y_{t}\right)_{t \in \mathbb{Z}}$ whose state space is $E=\left\{e_{1}, \ldots, e_{N-1}, \mathbf{0}\right\}$ and defined by (1) and (3). From the results of the previous subsection, we deduce the following corollary. $(\|\cdot\|$ denotes the Euclidian norm on $\mathbb{R}^{N-1}$.)

Corollary 2. Assume model (3) and let a function $g: E^{\mathbb{N}} \rightarrow \mathbb{R}^{N-1}$ be such that there exist a sequence $\left(\delta_{j}\right)_{j \in \mathbb{N}}$ which satisfies $\sum_{j \in \mathbb{N}} \sum_{k \geq j} \delta_{k}<\infty$ and

$$
\|g(x)-g(y)\| \leq \sum_{j \in \mathbb{N}} \delta_{j} \mathbb{1}_{x_{j} \neq y_{j}}
$$

Then, there exists a unique stochastic process $\left(Y_{t}\right)_{t \in \mathbb{Z}}$ taking values in $E$ such that

$$
\mathbb{P}\left(Y_{t}=e_{j} \mid \mathcal{F}_{t-1}\right)=\frac{\exp \left(g_{j}\left(Y_{t-1}, Y_{t-2}, \ldots\right)\right)}{1+\sum_{s=1}^{N-1} \exp \left(g_{s}\left(Y_{t-1}, Y_{t-2}, \ldots\right)\right)}, \quad 1 \leq j \leq N-1,
$$

where $g_{j}(\cdot)$ is the j'th component of $g$. Moreover $\left(Y_{t}\right)_{t \in \mathbb{Z}}$ is stationary and $\phi-$ mixing.

Proof. Denote by $p(\cdot \cdot)$ the probability kernel defined by

$$
p\left(e_{j} \mid x\right)=F_{j}\left[g\left(x_{0}, x_{1}, \ldots\right)\right], 1 \leq j \leq N-1,
$$

where $F_{j}: \mathbb{R}^{N-1} \rightarrow[0,1]$ is defined for $z \in \mathbb{R}^{N-1}$ by

$$
F_{j}(z)=\frac{\exp \left(z_{j}\right)}{1+\sum_{s=1}^{N-1} \exp \left(z_{s}\right)}, \quad 1 \leq j \leq N-1
$$

Because of (1), $F_{N}(z)=\left(1+\sum_{s=1}^{N-1} \exp \left(z_{s}\right)\right)^{-1}$. The Lipschitz assumption (5) implies that the $j$ 'th component of $g$ is bounded, for $j=1,2 \ldots, N-1$. Hence, there exists $\eta>0$ such that, for all $1 \leq j \leq N-1$ and $x \in E^{\mathbb{N}}$,

$$
\eta \leq p\left(e_{j} \mid x\right), \quad \eta \leq p(\mathbf{0} \mid x)
$$


Moreover, $F_{j}^{\prime}$ is bounded, for all $j$. Set $M=\max _{1 \leq j \leq N-1} \sup _{z \in \mathbb{R}^{N-1}}\left\|F_{j}^{\prime}(z)\right\|$. Then, if $x \stackrel{m}{=} y$ and $a \in E$, we have that

$$
\frac{p(a \mid x)}{p(a \mid y)} \geq 1-\frac{M \sum_{j \geq m+1} \delta_{j}}{\eta} .
$$

Provided that $m$ is large enough, choose $\gamma_{m}=M \sum_{j \geq m+1} \delta_{j} / \eta$. Hence, there exists an $m$ such that $\gamma_{m}=1-\eta$. Then we have $\sum_{k \geq 1} \gamma_{k}<\infty$ and using Remark 1, we have also $\sum_{k \geq 1} \gamma_{k}^{*}<\infty$. Then from Corollary 1 and Proposition 2, there exists a unique stationary solution $\left(Y_{t}\right)_{t \in \mathbb{Z}}$ satisfying (6) and the solution is $\phi$-mixing.

We note that the condition $\sum_{j \in \mathbb{N}} \sum_{k \geq j} \delta_{k}<\infty$ is equivalent to the condition $\sum_{j \in \mathbb{N}} j \delta_{j}<\infty$. For the infinite order linear model (4), Corollary 2 applies provided that $\sum_{j \geq 1} j\left\|A_{j}\right\|<\infty$ where $\left\|A_{j}\right\|$ denotes the corresponding operator norm of the matrix $A_{j}$. In particular, when $N=2$, we obtain that the logistic autoregressive model of infinite order is stationary and $\phi$-mixing if $\sum_{j \geq 1} j\left|A_{j}\right|<\infty$, where $\left(A_{j}\right)_{j \geq 1}$ denotes a real valued sequence. For the general non linear model defined in (3) but of finite-order, i.e. $g$ only depends on finitely many $y_{j}^{\prime}$ s, note that the assumptions of Corollary 2 are automatically satisfied because the number of coefficients $\delta_{j}$ is also finite.

\section{Categorical time series with a latent process}

In this section, we consider some specific instances of chains with complete connections. Following the methodology of GARCH models (see Engle (1982), Bollerslev (1986) and the text by Francq and Zakoïan (2010) for instance), and recalling the notation introduced in (3) we model the latent process $\left(\lambda_{t}\right)_{t \in \mathbb{Z}}$ to depend additionally on its past values. From a statistical perspective, such parametrization yields parsimony and allows for more flexible structures that can accommodate various forms of autocorrelation. To be specific, suppose that $p$ and $q$ are two positive integers and Let $f: \mathbb{R}^{(N-1) p} \times E^{q} \rightarrow \mathbb{R}^{N-1}$ be a function such that

$$
\lambda_{t}=f\left(\lambda_{t-1}, \ldots, \lambda_{t-p}, Y_{t-1}, \ldots, Y_{t-q}\right), \quad t \in \mathbb{Z}
$$

We will say that the process $\left(\left(Y_{t}, \lambda_{t}\right)\right)_{t \in \mathbb{Z}}$ is a solution of the problem $\mathcal{P}_{f}$ if (7) is satisfied and for each $t \in \mathbb{Z}$, $\lambda_{t}$ is $\mathcal{F}_{t-1}$-measurable. 


\subsection{A general result}

For $\underline{y} \in E^{q}$, define the mapping $G_{y}: \mathbb{R}^{(N-1) p} \rightarrow \mathbb{R}^{(N-1) p}$ by

$$
G_{\underline{y}}(\underline{x})=\left(f\left(x_{1}, \ldots, x_{p}, y_{1}, \ldots, y_{q}\right)^{\prime}, x_{1}^{\prime}, \ldots, x_{p-1}^{\prime}\right)^{\prime},
$$

where $f(\cdot)$ has been defined by (7). The main result of this section is the following.

Theorem 1. Suppose that there exist an integer $k \geq 1, \kappa \in(0,1)$ and $K>0$ such that

$$
\left\|G_{\underline{y}}(\underline{x})-G_{\underline{y}^{\prime}}\left(\underline{x}^{\prime}\right)\right\| \leq K\left(\left\|\underline{x}-\underline{x}^{\prime}\right\|+\mathbb{1}_{\underline{y} \neq \underline{y}^{\prime}}\right),
$$

and for all $\underline{x}, \underline{x}^{\prime}, \underline{y}_{1}, \ldots, \underline{y}_{k}$,

$$
\left\|G_{\underline{y}_{1}} \circ \cdots \circ G_{\underline{y}_{k}}(\underline{x})-G_{\underline{y}_{1}} \circ \cdots \circ G_{\underline{y}_{k}}\left(\underline{x}^{\prime}\right)\right\| \leq \kappa\left\|\underline{x}-\underline{x}^{\prime}\right\| .
$$

Then, the following hold true:

1. Let $\underline{x}$ be a vector of $\mathbb{R}^{(N-1) p}$ and $\left(\underline{y}_{j}\right)_{j \geq 1}$ a sequence of elements of $E^{q}$. Then the limit

$$
\lim _{s \rightarrow \infty} G_{\underline{y}_{1}} \circ \cdots \circ G_{\underline{y}_{s}}(x)
$$

exists and does not depend on $x$. Let $H:\left(E^{q}\right)^{\mathbb{N}} \rightarrow \mathbb{R}^{(N-1) p}$ be the function defined by

$$
H\left(\underline{y}_{1}, \underline{y}_{2}, \ldots\right)=\lim _{s \rightarrow \infty} G_{\underline{y}_{1}} \circ \cdots \circ G_{\underline{y}_{s}}(x) .
$$

Then the function $H$ is bounded. Moreover there exist $C>0$ such that

$$
\left\|H\left(\underline{y}_{1}, \underline{y}_{2}, \ldots\right)-H\left(\underline{y}_{1}^{\prime}, \underline{y}_{2}^{\prime}, \ldots\right)\right\| \leq C \sum_{j \geq 1} \kappa^{j / k} \mathbb{1}_{\underline{y}_{j} \neq \underline{y}_{j}^{\prime}}
$$

where $\mathrm{\kappa}$ is defined by (8).

2. A process $\left(\left(Y_{t}, \lambda_{t}\right)\right)_{t \in \mathbb{Z}}$ is solution of the problem $\mathcal{P}_{f}$ is and only if $\left(Y_{t}\right)_{t \in \mathbb{Z}}$ is a chain with complete connection associated to a function $g$ (see Corollary 2) defined by

$$
g\left(Y_{t-1}, Y_{t-2}, \ldots\right)=H_{1}\left(V_{t}, V_{t-1}, \ldots\right), \quad V_{t}=\left(Y_{t-1}, \ldots, Y_{t-q}\right) .
$$

Here $H_{1}$ denotes the $N-1$ first coordinates of the function $H$ defined previously.

3. There exists a unique strictly stationary solution to the equations (1) and (7). Moreover the process $\left(Y_{t}\right)_{t \in \mathbb{Z}}$ is $\phi$-mixing with geometrically decreasing mixing coefficients. This implies the ergodicity of the joint process $\left(\left(Y_{t}, \lambda_{t}\right)\right)_{t \in \mathbb{Z}}$. 
Proof. 1. The first part of the assertion is a straightforward consequence of the assumption and is omitted. We focus on the proof of the Lipschitz property of the function $H$. For $j \geq 1$, we set

$$
G_{\underline{y}}^{(j)}=G_{\underline{y}_{(j-1) k+1}} \circ G_{\underline{y}_{(j-1) k+2}} \circ \cdots \circ G_{\underline{y}_{j k}} .
$$

We have

$$
H\left(\underline{y}_{1}, \underline{y}_{2}, \ldots\right)=\lim _{s \rightarrow \infty} G_{\underline{y}}^{(1)} \circ \cdots \circ G_{\underline{y}}^{(s)}(x) .
$$

By the stated assumption, we obtain that

$$
\left\|G_{\underline{y}}^{(j)}(x)-G_{\underline{y}^{\prime}}^{(j)}(x)\right\| \leq \sum_{\ell=1}^{k} K^{\ell} \mathbb{1}_{\underline{y}_{(j-1) k+\ell}} \neq \underline{y}_{(j-1) k+\ell}^{\prime} .
$$

Hence

$$
\left\|G_{\underline{y}}^{(1)} \circ \cdots \circ G_{\underline{y}}^{(s)}(x)-G_{\underline{y}^{\prime}}^{(1)} \circ \cdots \circ G_{\underline{y}^{\prime}}^{(s)}(x)\right\| \leq \sum_{j=1}^{\infty} \kappa^{j} \sum_{\ell=1}^{k} K^{\ell} \mathbb{1}_{\underline{y}_{(j-1) k+\ell}} \underline{y}_{(j-1) k+\ell}^{\prime} .
$$

By setting $C=(K \vee 1)^{k}$ and letting $x \rightarrow \infty$ we obtain the result.

2. From the first point of the theorem, the necessary condition follows easily. Now let us assume that $\lambda_{t}=H_{1}\left(V_{t}, V_{t-1}, \ldots\right)$. Setting $\underline{\lambda}_{t}=H\left(V_{t}, V_{t-1}, \ldots\right)$, note that the continuity of the function $G_{V_{t}}$ implies that

$$
\begin{aligned}
\underline{\lambda}_{t} & =\lim _{s \rightarrow \infty} G_{V_{t}} \circ G_{V_{t-1}} \circ \cdots G_{V_{t-s}}(x) \\
& =G_{V_{t}}\left(\lim _{s \rightarrow \infty} G_{V_{t-1}} \circ \cdots \circ G_{V_{t-s}}(x)\right) \\
& =G_{V_{t}}\left(\underline{\lambda}_{t}\right),
\end{aligned}
$$

which proofs that $\left(\lambda_{t}\right)_{t \in \mathbb{Z}}$ satisfies (7).

3. The third point is a straightforward consequence of the two first results and of Corollary 2. Moreover the geometric decay of the $\phi$-mixing coefficients has been discussed in the remarks made following Proposition 2. Finally, it is well-known that $\phi$-mixing implies ergodicity of the process $\left(Y_{t}\right)_{t \in \mathbb{Z}}$ and then ergodicity of the process $\left(\left(\lambda_{t}, Y_{t}\right)\right)_{t \in \mathbb{Z}}$; see Samorodnitsky (2016, Ch. 2), for instance. 


\subsection{Linear models}

Let $A_{0}, A_{1}, \ldots, A_{p}, B_{1}, \ldots, B_{q}$ be some real matrices of size $(N-1) \times(N-1)$. We assume that

$$
f\left(x_{1}, \ldots, x_{p} ; y_{1}, \ldots, y_{q}\right)=A_{0}+\sum_{i=1}^{p} A_{i} x_{i}+\sum_{i=1}^{q} B_{i} y_{i} .
$$

Then the above model can be written alternatively as

$$
G_{V_{t}}(\underline{x})=\widetilde{A} \underline{x}+B
$$

with

$$
\widetilde{A}=\left(\begin{array}{cccc}
A_{1} & \cdots & A_{p-1} & A_{p} \\
I_{(N-1)(p-1)} & 0
\end{array}\right), \quad B=\left(\begin{array}{c}
A_{0}+\sum_{i=1}^{q} B_{i} Y_{t-i} \\
0 \\
\vdots \\
0
\end{array}\right),
$$

where $I_{(N-1)(p-1)}$ denotes the identity matrix of order $(N-1)(p-1)$. Then, the assumptions of Theorem 1 are satisfied if the spectral radius of $\widetilde{A}$ is less than unity (and then the norm of $\widetilde{A}^{k}$ is less than one if $k$ is large enough) which also implies that the roots of the polynomial $\mathcal{P}(z)=\operatorname{det}\left(I_{N-1}-\sum_{i=1}^{p} A_{i} z^{i}\right)$ are outside the unit disc; Lütkepohl (2005, Ch.2). For the case $p=q=1$, this result improves the conditions proved by Moysiadis and Fokianos (2014) since it does not require any additional assumption for the coefficient $B_{1}$. In fact, we reconfirm and generalize the condition obtained by Tjøstheim (2012) for the case of binary logistic autoregressive model when $p=q=1$. Compared with the work of Fokianos and Moysiadis (2017) we note again that for the case of logistic autoregressive modeling with binary data, these conditions simplify their findings.

\subsection{Non-linear models}

We discuss now the case of non-linear models. For example, a non-linear model that can be used for binary time series is a threshold type model. For a binary time series, we can define with some abuse of notation, the log-odds ratio by

$$
\lambda_{t}=d+\beta_{1} \lambda_{t-1}^{+}+\beta_{2} \lambda_{t-1}^{-}+\alpha Y_{t-1}
$$

where $x^{+}=\max (x, 0), x^{-}=\min (x, 0)$ and $\max \left\{\left|\beta_{1}\right|,\left|\beta_{2}\right|\right\}<1$. Such a model implies higher/lower probability of the occurrence of state 1 , depending on the sign of the lagged value of the latent process. Other models 
that can be introduced along the previous lines for binary time series are the so called smooth autoregressive models as advocated by Teräsvirta (1994). Such models will allow for smooth transitions between states.

Recall (7) and assume that there exists a norm $\|\cdot\|$ on $\mathbb{R}^{N-1}$ and some positive real numbers $\beta_{1}, \ldots, \beta_{p}, \alpha_{1}, \ldots, \alpha_{q}$ with $\alpha=\sum_{i=1}^{p} \alpha_{i}<1$ and for all $x_{1}, x_{1}^{\prime}, \ldots, x_{p}, x_{p}^{\prime}, y_{1}, y_{1}^{\prime}, \ldots, y_{q}, y_{q}^{\prime} \in \mathbb{R}^{N-1}$,

$$
\left\|f\left(x_{1}, \ldots, x_{p} ; y_{1}, \ldots, y_{q}\right)-f\left(x_{1}^{\prime}, \ldots, x_{p}^{\prime} ; y_{1}^{\prime}, \ldots, y_{q}^{\prime}\right)\right\| \leq \sum_{i=1}^{p} \alpha_{i}\left\|x_{i}-x_{i}^{\prime}\right\|+\sum_{i=1}^{q} \beta_{i}\left\|y_{i}-y_{i}^{\prime}\right\| .
$$

It can be proved under this condition that, for a process $\left(W_{t}\right)_{t \in \mathbb{Z}}$ defined by $W_{t}=\left(Y_{t-1}^{T}, \ldots, Y_{t-q}^{T}\right)^{T}$ and taking values in $\mathbb{R}^{(N-1) q}$, the random mapping

$$
G_{W_{t}}(x)=\left(f\left(x, W_{t}\right), x_{1}, \ldots, x_{p-1}\right)
$$

is contracting, after iteration. Indeed, if $x, x^{\prime} \in \mathbb{R}^{p}, \lambda_{i}^{x}=x_{i}$ for $1 \leq i \leq p$ and

$$
\lambda_{t}^{x}=f\left(\lambda_{t-1}^{x}, \ldots, \lambda_{t-p}^{x}, W_{t}\right), \quad t \geq p+1
$$

it follows by induction that

$$
\left\|\lambda_{t}^{x}-\lambda_{t}^{x^{\prime}}\right\| \leq \alpha^{\frac{t-p}{p}}\left\|x-x^{\prime}\right\|, \quad t \geq p+1
$$

Hence, there exists an integer $m \geq 1$, such that the mapping

$$
H_{t}^{(m)}(x)=G_{W_{t}} \circ G_{W_{t-1}} \circ \cdots \circ G_{W_{t-m}}(x)
$$

satisfies

$$
\left\|H_{t}^{(m)}(x)-H_{t}^{(m)}\left(x^{\prime}\right)\right\| \leq \kappa\left\|x-x^{\prime}\right\|
$$

for some $\kappa \in(0,1)$. Therefore the assumption of Theorem 1 is satisfied. We note again that this condition improves upon the conditions obtained by Moysiadis and Fokianos (2014) and Fokianos and Moysiadis (2017) since they require only that $\alpha<1$.

\section{Inclusion of exogenous covariates for finite-order Markov chain models}

In this section, we study the problem of including a covariate process $\left(Z_{t}\right)_{t \in \mathbb{Z}}$ in an autoregressive categorical time series model. We will be assuming that the covariate process is strongly exogenous and unbounded. We focus on the case of finite order Markov chains, i.e. the parameter $\lambda_{t}$ does not depend on its past values. 
The general case seems more difficult to tackle and will not be studied in the present paper; see Remark 5 for more.

We are not aware of any result which guarantees ergodicity of a model with covariate even in the simple case of a finite-state Markov chain. As we will see, there is an interesting parallel between Markov chains with exogenous covariates and Markov chains in random environments which have been studied in probability theory. In the proof of Theorem 2 given below, we will use an approach discussed in Cogburn (1984) for showing ergodicity of Markov processes in random environments.

\subsection{A general result for finite state Markov chains with covariates}

We will be discussing results concerning stationarity and ergodicity for a special case of time inhomogeneous Markov chain. We will consider a finite state Markov chain which can be jointly observed with a covariate process. In what follows, denote by $Z=\left(Z_{t}\right)_{t \in \mathbb{Z}}$ a stationary process with values in a measurable space $(G, \mathcal{G})$ and $\left(Y_{t}\right)_{t \in \mathbb{N}}$ a process which takes values in a finite set $E$. In addition, conditionally on $Z$, $\left(Y_{t}\right)_{t \in \mathbb{N}}$ is a finite-state inhomogeneous Markov chain. More precisely, we assume that there exist a family of transition matrices $\left\{P_{g}: g \in G\right\}$ such that

$$
\mathbb{P}\left(Y_{t}=y \mid Y_{t-1}=x ; Z\right)=P_{Z_{t}}(x, y), \quad(x, y) \in E^{2} .
$$

Throughout the section we will assume the following:

(E1) There exists an integer $m \geq 1$ such that for all $\left(z_{1}, z_{2}, \ldots, z_{m}\right) \in G^{m}$, the product of stochastic matrices $P_{z_{1}} P_{z_{2}} \cdots P_{z_{m}}$ has positive entries.

(E2) The process $Z$ is mixing in the ergodic theory sense, i.e for all elements $A$ and $B$ of $\mathcal{B}\left(G^{\mathbb{Z}}\right)$, we have

$$
\lim _{n \rightarrow \infty} \mathbb{P}\left(Z \in A, \tau^{n} Z \in B\right)=\mathbb{P}(Z \in A) \mathbb{P}(Z \in B),
$$

where $\tau$ denotes the shift operator on $F^{\mathbb{Z}}$ defined by $\tau Z=\left(Z_{j+1}\right)_{j \in \mathbb{Z}}$.

Remark 2. Note that Assumption (E1) implies that a process $\left(Y_{t}\right)_{t \in \mathbb{Z}}$ satisfying (9) also satisfies

$$
\mathbb{P}\left(Y_{t+m}=y \mid Y_{t}=x, Z\right)>0, \text { a.s } \quad(x, y, t) \in E \times E \times \mathbb{Z} .
$$

In addition, Assumption (E2) is stronger than assuming ergodicity of the process $Z$ but weaker than the classical strong mixing condition usually employed in the literature. A large number of useful stochastic processes are mixing, for instance the strong mixing processes and Bernoulli shifts defined by $Z_{t}=$ 
$H\left(\varepsilon_{t}, \varepsilon_{t-1}, \ldots\right)$ where $H$ is a measurable function and $\left(\varepsilon_{t}\right)_{t \in \mathbb{Z}}$ is an i.i.d sequence. Samorodnitsky (2016, Ch.2), discusses several properties of the different types of mixing in ergodic theory of stationary processes. Assumption (E2) will be employed for obtaining ergodicity for the shift operator $\tau^{m}$ which is not implied by the ergodicity of the shift operator $\tau$.

Remark 3. Model (9) implies an exogeneity assumption for the covariate process $\left(Z_{t}\right)_{t}$. In particular, for each time $t, Y_{t}$ is independent from $\left(Z_{s}\right)_{s \geq t+1}$ conditionally to $Y_{t-1}, Z_{t}, Y_{t-2}, Z_{t-1} \ldots$ which allows for simple computation of the conditional likelihood function. Indeed, we have for $y_{1}, \ldots, y_{n} \in E$,

$$
\mathbb{P}\left(Y_{2}=y_{2}, \ldots, Y_{n}=y_{n} \mid Y_{1}=y_{1}, Z_{1}, \ldots, Z_{n}\right)=\prod_{i=1}^{n} P_{Z_{i}}\left(y_{i-1}, y_{i}\right) .
$$

This type of exogeneity is also called Granger causality or Sims causality in the literature (see, for instance, Gourieroux and Monfort (1995, Sec. 1.5.2), for a discussion of these different concepts).

Remark 4. We can assume more generally that

$$
\mathbb{P}\left(Y_{t}=y \mid Z, Y_{t-1}=x\right)=P_{\widetilde{Z}_{t}}(x, y), \quad \widetilde{Z}_{t}=\left(Z_{t-j}\right)_{j \geq 0} .
$$

But this case is already covered by assumptions $(\mathbf{E 1})$ and $(\mathbf{E 2})$. Indeed, if the process $\left(Z_{t}\right)_{t}$ is assumed to be stationary and mixing, then the process $\left(\widetilde{Z}_{t}\right)_{t}$ taking values in $G^{\mathbb{Z}}$ is also stationary and mixing by using Samorodnitsky (2016, Prop. 2.2.4 and Cor. 2.2.5). Hence, if we assume (E1) for the covariate process $\left(\widetilde{Z}_{t}\right)_{t}$ taking values in $G^{\mathbb{Z}}$, assumption $(\mathbf{E 2})$ is automatically satisfied for the covariate process $\left(\widetilde{Z}_{t}\right)_{t}$ provided that $\left(Z_{t}\right)_{t}$ is mixing. In other words, there is no loss of generality by assuming that the timeinhomogeneous Markov chain only depends on the coordinate $Z_{t}$ of the covariate process. This point is important in applications because past values of covariates are routinely included in a regression model.

The main result of this section is given in the following theorem.

Theorem 2. Suppose that (E1-E2) hold true. Then there exists a unique stochastic process $\left(Y_{t}\right)_{t \in \mathbb{Z}}$ satisfying (9). Moreover the process $\left(\left(Y_{t}, Z_{t}\right)\right)_{t \in \mathbb{Z}}$ is ergodic.

Remark 5. Developing theory for models (3) and (7) which include exogenous covariates is a more challenging problem, as we explain next. Indeed, the proof of ergodicity relies heavily on assumption (E1) which asserts the positivity of the conditional density of the model we study. For simplicity, assume that $p=q=1$ and $N=2$ in (7) with

$$
\lambda_{t}=f\left(\lambda_{t-1}, Y_{t-1}, Z_{t}\right) .
$$


Then, the bivariate process $\left(\left(Y_{t}, \lambda_{t}\right)\right)_{t}$ is (conditionally on $\left.Z\right)$ a time-inhomogeneous Markov chain with transition kernel

$$
Q_{Z_{t}}((y, \lambda),\{1\} \times A)=F\left(f\left(\lambda, y, Z_{t}\right)\right) \delta_{f\left(\lambda, y, Z_{t}\right)}(A),
$$

where for $(a, z) \in \mathbb{R}^{2}, F(a)=\exp (a) /(1+\exp (a))$ and $\delta_{z}$ denotes the Dirac mass at point $z$. Therefore, the transition kernel $Q_{Z_{t}}$ is not absolutely continuous with respect to a measure which depends on $(\lambda, y)$. When there is no covariate, the Markov chain does not satisfy an irreducibility assumption and we recover a classical problem occurring to observation-driven time series models such as the Poisson GARCH type models; see for instance Fokianos et al. (2009), Neumann (2011) and Douc et al. (2013). In Section 3, we avoided this problem and proved ergodicity using a general coupling result for processes which are not necessarily Markov. If the dynamics of the model are specified conditionally to the realization of a covariate process, then a non-homogeneous version of this coupling technique might be possible to be derived for defining the conditional distribution of $Y \mid Z$ and then to prove existence of a stationary stochastic process $\left(\left(Y_{t}, Z_{t}\right)\right)_{t \in \mathbb{Z}}$ which satisfies the aforementioned recursions, by following the first part of the proof of Theorem 2. However, the probability kernel $Q$ does not have not a positive density w.r.t a dominating measure and therefore the second part of this proof cannot be adapted when covariates are included. In general, studying ergodicity properties of a stochastic process which includes simultaneously a latent process and a covariate process is more challenging problem and will be considered elsewhere.

Proof. We first show that the almost sure $\operatorname{limit} \lim _{s \rightarrow \infty} P_{Z_{t-s}} \cdots P_{Z_{t}}(x, y)$ exists for each $y \in E$ and does not depend on $x$. For Markov chains, this condition is comparable to the weak ergodicity notion, but here the limit is taken in the backward sense. See Seneta (2006) for several sufficient conditions ensuring weak ergodicity properties of time-inhomogeneous Markov chains, using ergodicity coefficients. Recall that the so-called Dobrushin's contraction coefficient of a stochastic matrix $P$ is defined by

$$
c(P)=\frac{1}{2} \sup _{x \neq y \in E}\|P(x, \cdot)-P(y, \cdot)\|_{T V}
$$

where for two probability measures $\mu$ and $v$ on the finite set $E$, the total variation distance between $\mu$ and $v$ is defined by $\|\mu-v\|_{T V}=\sum_{x \in E}|\mu(x)-v(x)|$. It is well known that we have the contraction

$$
\|\mu P-v P\|_{T V} \leq c(P)\|\mu-v\|_{T V}
$$

and for two stochastic matrices $P$ and $Q$, we have $c(P Q) \leq c(P) c(Q)$. 
Moreover $c(P) \leq 1-|E| \min _{x, y \in E} P(x, y)$, where $|E|$ denotes the cardinality of the set $E$. So Assumption (E1) ensures that for all $t \in \mathbb{Z}, c\left(P_{Z_{t}} P_{Z_{t+1}} \cdots P_{Z_{t+m-1}}\right)<1$ a.s. Now let $x \neq x^{\prime} \in E, t \in \mathbb{Z}$ and $s=k m+\ell$. we obtain by setting $\rho=1-\eta|E|$,

$$
\left\|P_{Z_{t-s+1}} \cdots P_{Z_{t}}(x, \cdot)-P_{Z_{t-s+1}} \cdots P_{Z_{t}}\left(x^{\prime}, \cdot\right)\right\|_{T V} \leq 2 c\left(P_{Z_{t-k m+1}} \cdots P_{Z_{t}}\right) \leq 2 \prod_{j=0}^{k-1} c\left(P_{Z_{t-(j+1) m+1}} \cdots P_{Z_{t-j m}}\right) .
$$

From Assumption (E2), the covariate process $Z$ is mixing. Then the process $\left(Z_{t-j}\right)_{j \in \mathbb{Z}}$ is also mixing. Indeed, if $\theta_{t}$ and $\tau$ denote the mappings defined on $G^{\mathbb{Z}}$ by $\theta_{t} x=\left(x_{t-i}\right)_{i \in \mathbb{Z}}$ and $\tau x=\left(x_{i+1}\right)_{i \in \mathbb{Z}}$ respectively, we have $\tau \circ \theta_{t}=\theta_{t} \circ \tau^{-1}$. Then for two Borel sets $A$ and $B$, we get

$$
\begin{aligned}
\mathbb{P}\left(\theta_{t} Z \in A, \tau^{n} \theta_{t} Z \in B\right) & =\mathbb{P}\left(Z \in \theta_{t}^{-1} A, \tau^{-n} Z \in \theta_{t}^{-1} B\right) \\
& =\mathbb{P}\left(\tau^{n} Z \in \theta_{t}^{-1} A, Z \in \theta_{t}^{-1} B\right) \\
& \rightarrow \mathbb{P}\left(\theta_{t} Z \in A\right) \mathbb{P}\left(\theta_{t} Z \in B\right)
\end{aligned}
$$

Moreover, observe that the operator $\tau^{m}$ is ergodic for $\mathbb{P}_{Z}$. Indeed, if a Borel set $A$ is such that $\tau^{m} A=A$, we obtain, using assumption E2,

$$
\mathbb{P}(Z \in A)=\mathbb{P}\left(\tau^{k m} Z \in A, Z \in A\right) \rightarrow_{k \rightarrow \infty} \mathbb{P}(Z \in A)^{2} .
$$

Then, we conclude that $\mathbb{P}_{Z}(A) \in\{0,1\}$, which shows that $\tau^{m}$ is ergodic. Now, using Assumption (E1), we have $\mathbb{E} \log c\left(P_{Z_{1}} \cdots P_{Z_{m}}\right)<0$. Then, from the ergodic theorem, we get

$$
\prod_{j=0}^{k-1} c\left(P_{Z_{t-(j+1) m+1}} \cdots P_{Z_{t-j m}}\right)=\exp \left(\sum_{j=0}^{k-1} \log c\left(P_{Z_{t-(j+1) m+1}} \cdots P_{Z_{t-j m}}\right)\right) \rightarrow_{k \rightarrow \infty} 0 .
$$

In addition, when $n \geq s$, we deduce that

$$
\left\|P_{Z_{t-s+1}} \cdots P_{Z_{t}}(x, \cdot)-P_{Z_{t-n}} \cdots P_{Z_{t}}(x, \cdot)\right\|_{T V} \leq 2 c\left(P_{Z_{t-s+1}} \cdots P_{Z_{t}}\right)
$$

From the Cauchy criterion, we deduce that the product of matrices $P_{Z_{t-s+1}} \cdots P_{Z_{t}}$ converges, when $s \rightarrow \infty$, to a stochastic matrix whose rows are all equal. Then there exists a measurable function $D: G^{\mathbb{N}} \rightarrow E^{N}$ with $N=|E|$ such that

$$
D\left(Z_{t}, Z_{t-1}, \ldots\right)=\lim _{s \rightarrow \infty} P_{Z_{t-s+1}} \cdots P_{Z_{t}}(x, \cdot) \text { a.s. }
$$

Setting $\bar{D}_{t}=D\left(Z_{t}, Z_{t-1}, \ldots\right), \bar{D}_{t}$ is a random probability measure on $E$. For $t \in \mathbb{Z}, z \in G^{\mathbb{Z}}, k$ a non-negative integer and $y_{0}, y_{1}, \ldots, y_{k} \in E$, we set

$$
\mu_{t: t+k}\left(z ; y_{0}, y_{1}, \ldots, y_{k}\right)=\prod_{i=1}^{k} P_{z_{t+i}}\left(y_{i-1}, y_{i}\right) \bar{D}_{t}\left(y_{0}\right) .
$$


From the Kolmogorov extension theorem, there exists for $\mathbb{P}_{Z}$-almost all values of $z \in \mathcal{B}\left(G^{\mathbb{Z}}\right)$ a unique measure $\mu(z, \cdot)$ on $E^{\mathbb{Z}}$ with marginals $\mu_{t: t+k}(z, \cdot)$. Hence, if $\zeta$ denotes the probability distribution of $Z$, the measure $\gamma$ defined by

$$
\gamma(A \times B)=\int_{B} \mu(z, A) \zeta(d z), \quad(A, B) \in \mathcal{B}\left(E^{\mathbb{Z}}\right) \times \mathcal{B}\left(F^{\mathbb{Z}}\right),
$$

is that of a couple from a stationary process $(Y, Z)$ satisfying $(9)$. To show uniqueness, let $\left(Y_{t}^{\prime}\right)_{t \in \mathbb{Z}}$ be another stochastic process satisfying (9). Then the distribution of $Y^{\prime} \mid Z=z$ is that of a non-homogeneous Markov chain with transitions $\left\{P_{z_{t}}: t \in \mathbb{Z}\right\}$. As shown before, this conditional distribution is unique and equal to $\mu(z, \cdot)$.

Next we show ergodicity of the process $\left(\left(Y_{t}, Z_{t}\right)\right)_{t \in \mathbb{Z}}$. To this end, we use an approach introduced in Cogburn (1984) for the study of Markov processes in random environment. This type of argument is also used in Sinn and Poupart (2011) for positive transition matrix $P_{Z_{t}}$ and we give here a more general and shorter proof. The approach used in Cogburn (1984) consists in considering the Markov kernel $Q$ on $E \times G^{\mathbb{Z}}$ defined by

$$
Q((x, z),\{y\} \times A)=P_{z_{1}}(x, y) \mathbb{1}_{A}(\tau z), \quad A \in \mathcal{B}\left(F^{\mathbb{Z}}\right) .
$$

If $v$ denotes the probability distribution $\left(Y_{t}, \tau^{t} Z\right)$ which takes values in $E \times G^{\mathbb{Z}}$, then $v$ is invariant for $Q$ and the process $\left(H_{t}\right)_{t \in \mathbb{Z}}$ defined by $H_{t}=\left(Y_{t}, \tau^{t} Z\right)$ is a Markov chain of transition kernel $Q$. Let $C$ be a $v-$ invariant set, i.e $Q((x, z), C)=1$ for $v$-almost every $(x, z) \in C$. Using Corollary 5.11 in Hairer (2006), the Markov chain $\left(H_{t}\right)_{t \in \mathbb{Z}}$ forms an ergodic process if and only if every $v$-invariant set $C$ is of measure 0 or 1 . In our case, we have $C=\cup_{x \in E}\{x\} \times C_{x}$ for some $C_{x} \in \mathcal{B}\left(F^{\mathbb{Z}}\right)$. To this end, we first note that if $C$ is $v$-invariant, then

$$
v(C)=v Q(C)=\int_{C} d v(x, z) Q((x, z), C)+\int_{C^{c}} d v(x, z) Q((x, z), C)=v(C)+\int_{C^{c}} d v(x, z) Q((x, z), C) .
$$

Then we get $Q((x, z), C)=0$ for $v$-almost every $(x, z) \in C^{c}$, the complement of $C$ in $E \times G^{\mathbb{Z}}$. Hence, we obtain $Q((x, z), C)=Q \mathbb{1}_{C}(x, z)=\mathbb{1}_{C}(x, z)$ for $v$-almost every $(x, z)$. But this also gives $Q^{m} \mathbb{1}_{C}=\mathbb{1}_{C}, v$ a.e, where $m$ has been defined by assumption (E1). Moreover, we have that

$$
Q^{m}((x, z), C)=\sum_{y \in E} \mathbb{1}_{C}\left(y, \tau^{m} z\right)\left[P_{z_{1}} \cdots P_{z_{m}}\right](x, y)
$$

We write $A=B v$-a.e. if $v(A \Delta B)=0$ where $A \Delta B$ denotes the symmetric difference of the sets $A$ and $B$, i.e $A \Delta B=\left(A \cap B^{c}\right) \cup\left(A^{c} \cap B\right)$. From assumption (E1), all the entries of the matrix $P_{z_{1}} \cdots P_{z_{m}}$ are positive. 
Then we deduce that for almost every $(x, z) \in C$, we have $\left(y, \tau^{m} z\right) \in C$ for all $y \in E$. We set $D=\cap_{y \in E} C_{y}$. Let us denote by $v_{1}$ and $v_{2}$ the marginals of $v$. We first note that for all $A \in \mathcal{B}\left(G^{\mathbb{Z}}\right)$, we have

$$
v(\{x\} \times A)=\int_{A} \mathbb{P}\left(Y_{0}=x \mid Z=z\right) v_{2}(d z)=\sum_{y \in E} \int_{A} \mathbb{P}\left(Y_{0}=x \mid Y_{-m}=y, Z=z\right) \mathbb{P}\left(Y_{n}=y \mid Z=z\right) v_{2}(d z) .
$$

We get

$$
v(\{x\} \times A) \geq \int_{A} \eta_{z} v_{2}(d z), \quad \eta_{z}=\min _{x, y \in E} \mathbb{P}\left(Y_{0}=x \mid Y_{-m}=y, Z=z\right) .
$$

Employing again assumption (E1), we have $\eta_{z}>0$ for all $z$ and we deduce that $v_{2}(A)=0$ as soon as $v(\{x\} \times A)=0$. Now for $x \in E$, we set $B_{x}=\tau^{m} C_{x} \backslash D$. As stated above we have

$$
v\left(\left\{(x, z): z \in B_{x}\right\}\right)=\sum_{x \in E} v\left(\{x\} \times B_{x}\right)=0 .
$$

We conclude that $v_{2}\left(B_{x}\right)=0$ for all $x \in E$ and then $v_{2}\left(\tau^{m} C_{x} \backslash C_{x}\right)=0$. By stationarity, $v_{2}\left(\tau^{m} C_{x}\right)=v_{2}\left(C_{x}\right)$. Therefore, for every $x \in E, \tau^{m} C_{x}=C_{x}$, $\mu$-a.e. But using assumption E2, we have that

$$
v_{2}\left(C_{x}\right)=v_{2}\left(\tau^{k m} C_{x} \cap C_{x}\right) \rightarrow_{k \rightarrow \infty} v_{2}\left(C_{x}\right)^{2}
$$

Then, we conclude that $v_{2}\left(C_{x}\right) \in\{0,1\}$. If $v_{2}\left(C_{x}\right)=0$ for every $x$, we easily get $v(C)=0$. Now if there exists $x \in E$ such that $v_{2}\left(C_{x}\right)=1$, we have, using the equality $v_{2}\left(B_{x}\right)=0$,

$$
1 \leq v_{2}\left(C_{x}\right)=v_{2}\left(\tau^{m} C_{x}\right)=v_{2}\left(\tau^{m} C_{x} \cap D\right) \leq v_{2}(D) \leq \min _{y \in E} v_{2}\left(C_{y}\right)
$$

Then $v_{2}\left(C_{y}\right)=1$ for each $y \in E$. Finally we obtain

$$
v(C)=\sum_{y \in E} v\left(\{y\} \times C_{y}\right)=\sum_{y \in E} v_{1}(y)=1 .
$$

Hence, we have shown that the process $\left(H_{t}\right)_{t \in \mathbb{Z}}$ is ergodic and so is the process $\left(\left(Y_{t}, Z_{t}\right)_{t \in \mathbb{Z}}\right.$.

\subsection{Application to the multinomial logistic model with covariates}

We assume here that conditionally to a covariate process $\left(Z_{t}\right)_{t \in \mathbb{Z}}$ taking values in $\mathbb{R}^{d}$, the process $\left(Y_{t}\right)_{t \in \mathbb{Z}}$ is a $q$-order Markov chain such that

$$
\mathbb{P}\left(Y_{t}=e_{j} \mid Y_{t-1}, \ldots, Y_{t-q}, Z\right)=\frac{\exp \left(g_{j}\left(Y_{t-1}, \ldots, Y_{t-q} ; Z_{t}\right)\right)}{1+\sum_{s=1}^{N-1} \exp \left(g_{s}\left(Y_{t-1}, \ldots, Y_{t-q} ; Z_{t}\right)\right)}:=Q_{Z_{t}}\left(Y_{t-q: t-1}, e_{j}\right)
$$


for some measurable functions $g_{j}: E^{q} \times \mathbb{R}^{d}, 1 \leq j \leq N-1$. Let us check that assumption $\mathbf{E} 1$ is satisfied for the conditional Markov chain $\left(X_{t}\right)_{t \in \mathbb{Z}}$ defined by $X_{t}=\left(Y_{t}^{T}, Y_{t-1}^{T}, \ldots, Y_{t-q+1}^{T}\right)^{T}$. Conditionally to $Z$, the process $\left(X_{t}\right)_{t \in \mathbb{Z}}$ defines a time-inhomogeneous Markov chains such that

$$
P_{Z_{t}}\left(\left(u_{1}, \ldots, u_{q}\right),\left(v_{1}, \ldots, v_{q}\right)\right):=Q_{Z_{t}}\left(\left(u_{1}, \ldots, u_{q}\right), v_{1}\right) \prod_{s=1}^{q-1} \mathbb{1}_{v_{s+1}=u_{s}} .
$$

Since the transition $Q_{Z_{t}}$ takes only positive values, the assumption E1 follows by taking $m=q$. Then assuming $\mathbf{E 2}$ for the covariate process, Theorem 2 applies and guarantees the ergodicity of the process $\left(\left(Y_{t}, Z_{t}\right)\right)_{t \in \mathbb{Z}}$. These results show that our approach simplifies conditions required to obtain consistency and asymptotic normality of the maximum likelihood estimator, even in the case of considering covariates. However, existence of moments for the covariate process is still required to study large sample properties the maximum likelihood estimator.

Comparing these results with the work of Kaufmann (1987), we note that likelihood inference can be developed without making assumptions on the design of covariates and assuming that they are bounded. The work by Kaufmann (1987) did not study ergodicity properties of categorical time series models with covariates though. Additional previous work by Kedem and Fokianos (2002) on likelihood estimation employs an assumption regarding ergodicity of the joint process $\left(Y_{t}^{T}, Z_{t}^{T}\right)^{T}$ (see Assumption A in Kedem and Fokianos (2002, pp.16-17)). Theorem 2 shows that such assumptions are not necessary, at least in the context of categorical time series models. Finally, comparing our work with that of de Jong and Woutersen (2011) who consider the case of a probit model for binary time series we see that the results we obtain apply in this case without assuming an alpha-mixing condition on the covariate process.

\section{Acknowledgements}

Part of this work was done while K. Fokianos was with the Department of Mathematics \& Statistics, University of Cyprus. The authors thank the Editor, the Associate Editor and a referee for several useful comments and suggestions. 


\section{References}

Biswas, A. and P. X.-K. Song (2009). Discrete-valued ARMA processes. Statistics $\mathcal{E}$ Probability Letters 79, $1884-1889$.

Bollerslev, T. (1986). Generalized autoregressive conditional heteroskedasticity. Journal of Econometrics 31, 307-327.

Bradley, R. (2007). Introduction to Strong Mixing Conditions. Vol. 1. Kendrick Press, Heber City, UT.

Breen, W., L. R. Glosten, and R. Jagannathan (1989). Economic significance of predictable variations in stock index returns. The Journal of Finance 44, 1177-1189.

Bressaud, X., R. Fernández, and A. Galves (1999). Decay of correlations for non-Hölderian dynamics. a coupling approach. Electron. J. Probab. 4, 1-19.

Butler, K. C. and S. Malaikah (1992). Efficiency and inefficiency in thinly traded stock markets: Kuwait and Saudi Arabia. Journal of Banking \& Finance 16, 197-210.

Christoffersen, P., F. X. Diebold, R. S. Mariano, A. Tay, and Y. K. Tse (2007). Direction-of-change forecasts for asian equity markets based on conditional variance, skewness and kurtosis dynamics: Evidence from hong kong and singapore. Journal of Financial Forecasting 1, 1-22.

Christoffersen, P. F. and F. X. Diebold (2006). Financial asset returns, direction-of-change forecasting, and volatility dynamics. Management Science 52, 1273-1287.

Cogburn, R. (1984). The ergodic theory of Markov chains in randon environments. Z. Wahrscheinlichkeitstheory verw. Gebiete 66, 109-128.

Cox, D. R. (1981). Statistical analysis of time series: some recent developments. Scand. J. Statist. 8, 93-115.

Cox, D. R. and E. J. Snell (1970). The Analysis of Binary Data. London: Chapman \& Hall.

de Jong, R. M. and T. Woutersen (2011). Dynamic time series binary choice. Econometric Theory 27, $673-702$.

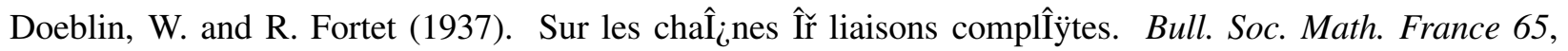
$132-148$. 
Douc, R., P. Doukhan, and E. Moulines (2013). Ergodicity of observation-driven time series models and consistency of the maximum-likelihood estimator. Stochastic Processes and their Applications 123, 26202647.

Doukhan, P. (1994). Mixing: properties and examples. Number 85 in Lecture Notes in Statistics. New York: Springer-Verlag.

Doukhan, P., K. Fokianos, and D. Tjøstheim (2012). On weak dependence conditions for Poisson autoregressions. Statist. Probab. Lett. 82, 942-948.

Engle, R. F. (1982). Autoregressive conditional heteroscedasticity with estimates of the variance of United Kingdom inflation. Econometrica 50, 987-1007.

Fahrmeir, L. and H. Kaufmann (1987). Regression models for nonstationary categorical time series. Journal of Time Series Analysis 8, 147-160.

Fahrmeir, L. and G. Tutz (2001). Multivariate statistical modelling based on generalized linear models (Second ed.). Springer Series in Statistics. New York: Springer-Verlag. With contributions by Wolfgang Hennevogl.

Fokianos, K. and B. Kedem (2003). Regression theory for categorical time series. Statist. Sci. 18, 357-376.

Fokianos, K. and T. Moysiadis (2017). Binary time series friven by a latent process. Econometrics and Statistics 2, 117-130.

Fokianos, K., A. Rahbek, and D. Tjostheim (2009). Poisson autoregression. J. Amer. Statist. Assoc. 104, $1430-1439$.

Fokianos, K. and D. Tjøstheim (2011). Log-linear Poisson autoregression. J. Multivariate Anal. 102, 563578.

Francq, C. and J.-M. Zakoïan (2010). GARCH models: Stracture, Statistical Inference and Financial Applications. United Kingdom: Wiley.

Gourieroux, C. and A. Monfort (1995). Statistics and Econometric Models. Volume 1. Cambridge University Press. 
Hairer, M. (2006). Ergodic properties of Markov processes. Lecture notes available at http: //www . hairer . org/notes/Markov .pdf.

Harris, T. (1955). On chains of infinite order. Pacific J. Math. 5, 707-724.

Iosifescu, M. and S. Grigorescu (1990). Dependence with Complete Connections and its Applications. Cambridge University Press.

Joe, H. (1997). Multivariate Models and Dependence Concepts. London: Chapman \& Hall.

Kaufmann, H. (1987). Regression models for nonstationary categorical time series: Asymptotic estimation theory. Annals of Statistics 15, 79-98.

Kauppi, H. (2012). Predicting the direction of the Fed's target rate. Journal of Forecasting 31, 47-67.

Kauppi, H. and P. Saikkonen (2008). Predicting US recessions with dynamic binary response models. The Review of Economics and Statistics 90, 777-791.

Kedem, B. (1980). Binary Time Series. Marcel Dekker, New York.

Kedem, B. and K. Fokianos (2002). Regression models for time series analysis. Hoboken, NJ: Wiley.

Lütkepohl, H. (2005). New Introduction to Multiple Time Series Analysis (1st ed.). Berlin: Springer.

MacDonald, I. L. and W. Zucchini (1997). Hidden Markov and Other Models for Discrete-valued Time Series. London: Chapman \& Hall.

McCullagh, P. and J. A. Nelder (1989). Generalized Linear Models (2nd ed.). London: Chapman \& Hall.

Moysiadis, T. and K. Fokianos (2014). On binary and categorical time series models with feedback. J. Multivariate Anal. 131, 209-228.

Neumann, M. (2011). Absolute regularity and ergodicity of poisson count processes. Bernoulli 17, 12681284.

Nyberg, H. (2010). Dynamic probit models and financial variables in recession forecasting. Journal of Forecasting 29, 215-230.

Nyberg, H. (2011). Forecasting the direction of the us stock market with dynamic binary probit models. International Journal of Forecasting 27, 561-578. 
Nyberg, H. (2013). Predicting bear and bull stock markets with dynamic binary time series models. Journal of Banking E Finance 37, 3351-3363.

Pamminger, C. and S. Frühwirth-Schnatter (2010). Model-based clustering of categorical time series. Bayesian Anal. 5, 345-368.

Qaqish, B. F. (2003). A family of multivariate binary distributions for simulating correlated binary variables with specified marginal means and correlations. Biometrika 90, 455-463.

Russell, J. R. and R. F. Engle (1998). Econometric Analysis of Discrete-Valued Irregularly-Spaced Financial Transactions Data Using a New Autoregressive Conditional Multinomial Model. SSRN eLibrary.

Russell, J. R. and R. F. Engle (2005). A discrete-state continuous-time model of financial transactions prices and times. Journal of Business and Economic Statistics 23, 166-180.

Rydberg, T. H. and N. Shephard (2003). Dynamics of trade-by-trade price movements: decomposition and models. Journal of Financial Econometrics 1, 2-25.

Samorodnitsky, G. (2016). Stochastic Processes and Long Range Dependence. Springer.

Seneta, E. (2006). Non-negative matrices and Markov chains. Springer Series in Statistics. Springer, New York.

Sinn, M. and P. Poupart (2011). Asymptotic theory for linear-chain conditional random fields. In G. Gordon, D. Dunson, and M. Dudik (Eds.), Proceedings of the Fourteenth International Conference on Artificial Intelligence and Statistics, Volume 15 of Proceedings of Machine Learning Research, Fort Lauderdale, FL, USA, pp. 679-687.

Slud, E. V. and B. Kedem (1994). Partial likelihood analysis of logistic regression and autoregression. Statist. Sinica 4, 89-106.

Startz, R. (2008). Binomial autoregressive moving average models with an application to US recessions. Journal of Business E Economic statistics 26, 1-8.

Stern, R. D. and R. Coe (1984). A model fitting analysis of daily rainfall data. Journal of the Royal Statistical Society. Series A 147, pp. 1-34. 
Teräsvirta, T. (1994). Specification, estimation, and evaluation of smooth transition autoregressive models. Journal of the American Statistical Association 89, 208-218.

Tjøstheim, D. (2012). Rejoinder on: Some recent theory for autoregressive count time series. TEST 21, $469-476$.

Weiß, C. (2011). Generalized choice models for categorical time series. J. Statist. Plann. Inference 141, $2849-2862$.

Wu, R. and Y. Cui (2014). A parameter-driven logit regression model for binary time series. Journal of Time Series Analysis 35, 462-477.

Zeger, S. L. and B. Qaqish (1988). Markov regression models for time series: a quasi-likelihood approach. Biometrics 44, 1019-1031. 